\title{
Quality of Life Improves with Alemtuzumab Over 6 Years in Relapsing-Remitting Multiple Sclerosis Patients with or without Autoimmune Thyroid Adverse Events: Post Hoc Analysis of the CARE-MS Studies
}

Antonio Bertolotto · Rafael Arroyo · Elisabeth G. Celius · Giancarlo Comi · Eva Kubala Havrdova

William David Honeycutt · Samuel F. Hunter · Guillermo Izquierdo · Barbara Kornek ·

Tamara Miller · Dimos D. Mitsikostas • Barry A. Singer · Tjalf Ziemssen • Luke Chung •

Nadia Daizadeh $\cdot$ Salman Afsar $\cdot$ Lobat Hashemi $\cdot$ Peter Senior

Received: December 11, 2019 / Published online: May 14, 2020

(c) The Author(s) 2020

\section{ABSTRACT}

Introduction: In clinical trials of alemtuzumab, autoimmune thyroid adverse events (AEs) were frequent. Here, we assess the impact of thyroid AEs on health-related quality of life (HRQL) in

Digital features To view digital features for this article go to https://doi.org/10.6084/m9.figshare.12229493.

Electronic supplementary material The online version of this article (https://doi.org/10.1007/s40120020-00191-7) contains supplementary material, which is available to authorized users.

\section{A. Bertolotto $(\square)$}

SCDO Neurologia-CRESM (Centro Riferimento Regionale Sclerosi Multipla), University Hospital San Luigi Gonzaga, Orbassano, Turin, Italy e-mail: antonio.bertolotto@gmail.com

R. Arroyo

Hospital Universitario Quirónsalud Madrid, Madrid, Spain

E. G. Celius

Oslo University Hospital Ullevål and Institute of Clinical Medicine, University of Oslo, Oslo, Norway

G. Comi

University Vita-Salute San Raffaele, Milan, Italy

E. K. Havrdova

First Medical Faculty, Charles University, Prague,

Czech Republic alemtuzumab-treated patients with relapsingremitting multiple sclerosis (RRMS).

Methods: In phase 3 CARE-MS I (NCT00530348) and II (NCT00548405) trials, patients with RRMS were administered alemtuzumab $12 \mathrm{mg} /$ day on 5 consecutive days at baseline and on 3 consecutive days 12 months later. Patients could participate in an extension study (NCT00930553) through year 6. HRQL was assessed at baseline and annually using the Functional Assessment of Multiple Sclerosis (FAMS), EuroQoL-5 Dimension Visual Analog Scale (EQ-5D VAS), and 36-Item Short-Form Survey (SF-36) questionnaires. Outcomes were

\section{W. D. Honeycutt}

Neurology Associates, Maitland, FL, USA

\section{S. F. Hunter}

Advanced Neurosciences Institute, Franklin, TN, USA

G. Izquierdo

Vithas Nisa Hospital, Seville, Spain

B. Kornek

Medical Neuroscience Cluster, Medical University of Vienna, Vienna, Austria

T. Miller

Advanced Neurology of Colorado, Fort Collins, CO, USA 
analyzed in patients with or without thyroid AEs (nonserious or serious). A subset of patients with thyroid AEs was analyzed to assess HRQL before and during the onset of thyroid AEs.

Results: A total of 811 CARE-MS patients were treated with alemtuzumab. Of these, 342 (42\%) patients experienced thyroid AEs over 6 years; serious thyroid AEs occurred in 44 (5\%) patients. At year 6, HRQL outcomes generally remained slightly improved or similar to core study baseline in alemtuzumab-treated patients with or without thyroid AEs: FAMS (leastsquares mean change from baseline without thyroid AEs, 0.7; with nonserious thyroid AEs, 5.1; with serious thyroid AEs, - 5.3), EQ-5D VAS $(2.0 ; 3.0 ;-6.8)$, SF-36 mental component summary (MCS $[0.6 ; 1.6 ;-2.8])$, SF-36 physical component summary (PCS $[0.8 ; 1.0 ; 1.1])$. Over 6 years, $63-82 \%$ of patients in each group had improved/stable SF-36 MCS and PCS scores. Among patients with thyroid AE onset in year 3 (peak incidence), there were minimal differences between HRQL outcomes before onset (year 2) and after onset (year 3).

Conclusion: Autoimmune thyroid AEs (serious and nonserious) had minimal impact on HRQL in alemtuzumab-treated patients. These data may aid therapeutic decisions in patients with relapsing MS.

D. D. Mitsikostas

1st Neurology Department, Aeginition Hospital, National and Kapodistrian University of Athens, Athens, Greece

B. A. Singer

MS Center for Innovations in Care, Missouri Baptist

Medical Center, St Louis, MO, USA

T. Ziemssen

Center of Clinical Neuroscience, Carl Gustav Carus

University Hospital, Dresden, Germany

L. Chung · N. Daizadeh · S. Afsar · L. Hashemi

Sanofi, Cambridge, MA, USA

P. Senior

University of Alberta, Edmonton, AB, Canada

\section{PLAIN LANGUAGE SUMMARY}

This study looked at alemtuzumab, an approved treatment for multiple sclerosis (MS). People who receive alemtuzumab may develop thyroid problems. The researchers wanted to know whether people who developed thyroid problems with alemtuzumab had a worse quality of life compared with those who did not. The researchers measured quality of life using a questionnaire. The questionnaire looked at people's physical, social, and psychological well-being over 6 years. A total of 811 people with MS treated with alemtuzumab took part in this study. Of these, 469 people (58\%) did not develop thyroid problems and 342 people $(42 \%)$ developed thyroid problems. The thyroid problems were serious in 44 people. The researchers observed that thyroid problems during alemtuzumab treatment did not make quality of life worse in most people. Some people with serious thyroid problems had worsened quality of life; this was mostly among people who required certain treatments for their thyroid problems. Quality of life did not change much in people while the thyroid problems were ongoing. This study shows that thyroid problems after alemtuzumab treatment for MS have little negative impact on quality of life for most people. These findings may help healthcare providers make decisions about MS treatment.

Keywords: Alemtuzumab; Health-related quality of life; Thyroid adverse events; Relapsing-remitting multiple sclerosis 


\section{Key Summary Points}

\section{Why carry out this study?}

Alemtuzumab is an approved diseasemodifying therapy for the treatment of multiple sclerosis (MS) that improves health-related quality of life (HRQL) in patients with MS, but also carries a risk for autoimmune adverse events (AEs), mainly affecting the thyroid.

The aim of the study was to evaluate the impact of autoimmune thyroid AEs on HRQL in alemtuzumab-treated patients.

\section{What was learned from the study?}

Over 6 years, HRQL outcomes generally remained slightly improved or similar to baseline in most patients who developed thyroid AEs after initiating alemtuzumab.

In patients with serious thyroid AEs, transient worsening in HRQL was associated with treatment required for thyroid AEs (thyroidectomy or radioiodine ablation).

These findings reflect the patientperceived therapeutic benefit of alemtuzumab despite the occurrence of thyroid AEs, and thus can aid therapeutic decisions in patients with relapsing MS.

\section{INTRODUCTION}

Multiple sclerosis (MS) is a chronic, autoimmune, neurodegenerative disease that reduces health-related quality of life (HRQL) in affected individuals and impairs activities of daily living. Disease-modifying therapies (DMTs) for MS aid in symptom management and slow the course of disease, and can thus improve HRQL in patients with MS [1]; however, because all DMTs carry risks for unwanted side effects, the net impact of a DMT on HRQL reflects therapeutic efficacy as well as adverse events (AEs) [2].
Alemtuzumab (Lemtrada ${ }^{\circledR}$, Sanofi Genzyme, Cambridge, MA) is a humanized anti-CD52 monoclonal antibody indicated for patients with relapsing forms of MS. The efficacy and safety of alemtuzumab were evaluated in the 2-year, phase 3 CARE-MS I (NCT00530348) and II (NCT00548405) trials, and for an additional 4 years in the CAMMS03409 extension study (NCT00930553) [3-6]. Alemtuzumab significantly reduced relapses, disability, magnetic resonance imaging (MRI) lesions, and brain volume loss compared with subcutaneously administered interferon beta-1a (SC IFNB-1a) in the core studies, and alemtuzumab efficacy was maintained through year 6 . In parallel with these clinical and MRI improvements, alemtuzumab-treated patients reported significantly greater HRQL improvements from baseline to year 2 compared with SC IFNB-1a-treated patients, and HRQL with alemtuzumab remained above baseline or similar to baseline through year 6 [7-9].

AEs associated with alemtuzumab in clinical trials and postmarketing experience include infusion-associated reactions, increased frequency of infection, and the potential for opportunistic infections, secondary autoimmunity (thyroid disorders, immune thrombocytopenia, nephropathies, autoimmune cytopenias, autoimmune hepatitis, and other less common autoimmune events), acute acalculous cholecystitis, and cardiovascular and pulmonary events possibly related to infusion [3, 4, 10-13]. Thyroid AEs were the most frequent autoimmune AEs associated with alemtuzumab. In the phase 3 alemtuzumab trials and their extension study, the overall rate of thyroid AEs was $42 \%$ over 6 years, peaking in year 3 following the first alemtuzumab course, which was received at baseline, and declining thereafter [3-6].

Information is currently lacking on the consequences of thyroid AEs on patients' lives after treatment with alemtuzumab. The purpose of this study was to evaluate the impact of autoimmune thyroid AEs on HRQL in alemtuzumab-treated patients from the CARE-MS studies. 


\section{METHODS}

\section{Patients and Procedures}

CARE-MS I and II were randomized, rater-blinded, active-controlled, head-to-head trials of alemtuzumab (12 mg/day on 5 consecutive days at baseline and on 3 consecutive days 12 months later) compared with SC IFNB-1a (44 $\mu$ g three times/week) $[3,4]$. In the CARE-MS extension study, patients randomized to alemtuzumab could receive additional courses $(12 \mathrm{mg} /$ day on 3 consecutive days at least 12 months after the previous alemtuzumab course) as needed for disease activity [10, 12]. Patients could also receive other licensed DMTs at the trial investigator's discretion (Fig. 1). The CARE-MS extension study was carried out in 178 study centers in 23 countries.

In alemtuzumab-treated patients, thyroid function testing was carried out through a comprehensive monitoring program. Thyroid AEs listed in this study were reported as AEs by the investigators, and did not include patients with thyroid events reported as lab abnormalities but not reported as AEs $(n=25)$. Thyroid- stimulating hormone (TSH) was measured at baseline and quarterly until 48 months after the last alemtuzumab infusion. In the event of abnormal TSH levels (normal range, 0.4-4.0 mU/L), further investigation was done by assessing thyroid symptoms (hyperthyroidism or hypothyroidism), free T3, free T4, and thyroid autoantibodies at the investigator's discretion [14-16]. Serious thyroid AEs were defined as those that were life threatening, required hospitalization, or resulted in persistent or significant disability, congenital anomaly, or death. Nonserious thyroid AEs were those that did not meet the criteria for serious thyroid AEs.

Thyroid AE severity was evaluated using the National Cancer Institute Common Terminology Criteria for Adverse Events (CTCAE) version 3.0 [17]: grade 1 AEs are mild or asymptomatic, and no treatment is necessary; grade 2 AEs are moderate and symptomatic, but do not interfere with daily activities, and noninvasive intervention is indicated (e.g., thyroid suppression therapy for hyperthyroidism or thyroid replacement for hypothyroidism); grade 3 AEs are severe, requiring hospitalization or prolongation of hospitalization; grade 4 AEs are life

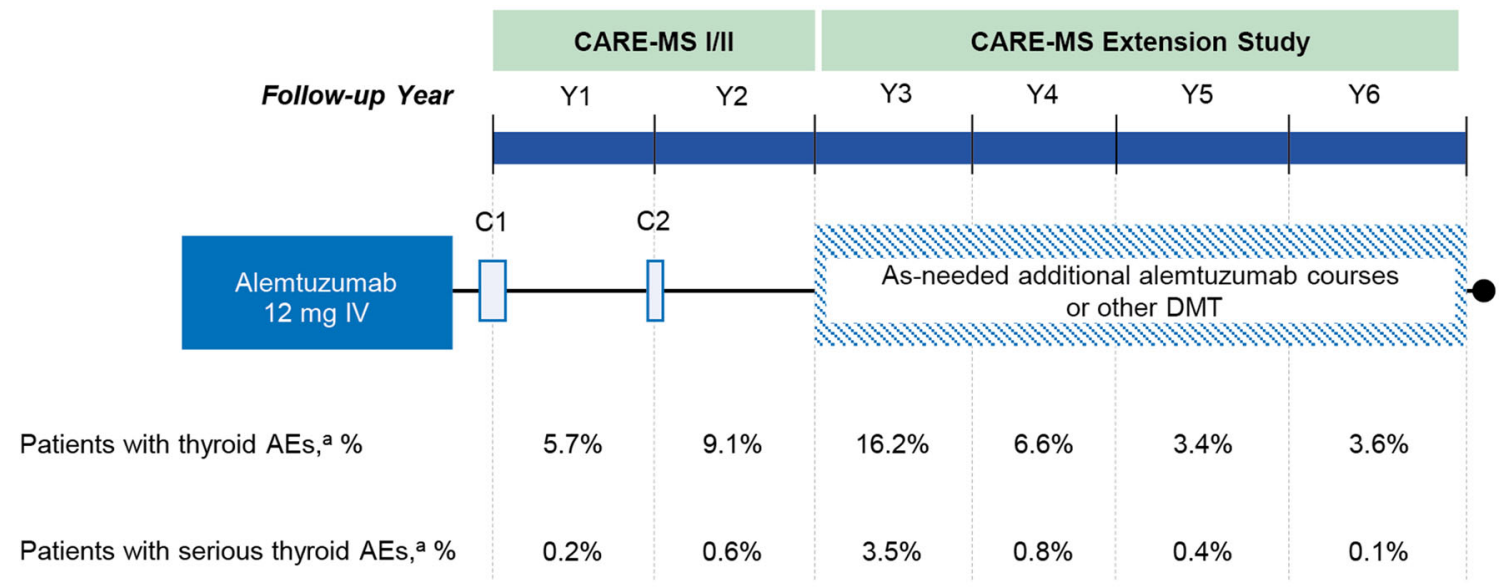

Fig. 1 Designs of the CARE-MS I/II and CARE-MS extension studies, including timing of alemtuzumab dosing and annual incidence of thyroid AEs. Patients randomized to alemtuzumab in the core CARE-MS I/II trials received treatment on 5 consecutive days at baseline and on 3 consecutive days 12 months later. In the CARE-MS extension (CAMMS03409) study, patients could receive additional courses of alemtuzumab ( $12 \mathrm{mg} /$ day on 3 consecutive days at least 12 months after the previous course) as needed for predefined disease activity at the investigator's discretion; other approved DMTs were also permitted. ${ }^{a}$ First occurrence of thyroid AE for a patient. AE adverse event, C course, DMT disease-modifying therapy, IV intravenous, $\mathrm{Y}$ year 
threatening or disabling and require urgent intervention; grade 5 AEs are fatal.

\section{HRQL Assessments}

HRQL questionnaires were administered to patients at baseline and every year, and included the Functional Assessment of Multiple Sclerosis (FAMS), Quality of Life-5 Dimension Visual Analog Scale (EQ-5D VAS), and 36-Item Short-Form Survey (SF-36).

FAMS version 4 is a reliable, MS-specific questionnaire consisting of 44 scored items in six clinically relevant domains including mobility, symptoms, emotional well-being, general contentment, thinking/fatigue, and family/social well-being. FAMS (total) scores range from 0 to 176 [18]. The EQ-5D VAS is a validated, generic HRQL measure consisting of a single-item scale comprising five dimensions: mobility, self-care, usual activities, pain/discomfort, and anxiety/depression. EQ-5D VAS scores range from 0 to 100 [19]. SF-36 version 2 is a widely used generic measure of a patient's state of health, comprising 36 items organized into eight scales and combined into two summary measures: the mental component summary (MCS) and physical component summary (PCS) survey [20]. The SF-36 utilizes a normbased scoring method in which scores have a mean of 50 and standard deviation of 10 in healthy populations [21]. Clinically meaningful worsening and improvement on the SF-36 are defined as at least a 5-point decrease and increase from baseline norm-based scores, respectively [22]. The 5-point cutoff is based on half a standard deviation, which represents a minimally important difference. Higher scores in all of the above mentioned questionnaires represent better HRQL.

\section{Statistical Analysis}

Thyroid AE incidences were based on the first occurrence of a thyroid AE to avoid double counting an ongoing event. Patients were stratified initially into subgroups on the basis of the presence or absence of autoimmune thyroid AEs, then further stratified on the basis of the presence of nonserious and serious thyroid AEs. Changes in HRQL outcomes from core study baseline and group differences at each time point were estimated by least-squares mean and 95\% confidence interval, using a mixed-effect repeated measure model, with unstructured covariance model with a time-by-group interaction and covariate adjustment for group, time, geographic region, and baseline score. No imputation was made for patients with missing HRQL outcomes at any postbaseline time points, due to the high patient retention rate. The subset of alemtuzumab-treated CARE-MS patients with thyroid $\mathrm{AE}$ onset during year 3 (i.e., third year after receiving the first alemtuzumab course at baseline) was also analyzed separately to investigate HRQL outcomes before (year 2) and during/after onset (year 3).

\section{Compliance with Ethics Guidelines}

The CARE-MS I and II studies and the CARE-MS extension study were registered with ClinicalTrials.gov (NCT00530348, NCT00548405, and NCT00930553, respectively). The studies were conducted in accordance with the ethical principles outlined in the Declaration of Helsinki. All procedures were approved by local institutional ethics review boards of participating sites (see Table $\mathrm{S} 1$ in the electronic supplementary material for details). Patients provided written informed consent.

\section{RESULTS}

\section{Autoimmune Thyroid AEs and Patient Baseline Characteristics}

A total of 811 patients were treated with alemtuzumab $12 \mathrm{mg}$ in the CARE-MS I $(n=376)$ and II $(n=435)$ trials. Of these, $742(91 \%)$ patients entered the extension and 659 (81\%) remained on study from core study baseline to year 6 post alemtuzumab initiation. Forty-eight (6\%) patients completed the core studies and did not enroll in the extension, and 104 (13\%) discontinued from the core or extension studies (due to withdrawal of consent [7.3\%], lost to follow- 
Table 1 Incidence of autoimmune thyroid AEs

\begin{tabular}{|c|c|c|c|c|c|c|c|}
\hline & \multicolumn{6}{|c|}{ Incidence, $n(\%)$} & \multirow{2}{*}{$\begin{array}{l}\text { EAIR per } 100 \\
\text { patient-years }^{\text {a }} \\
\text { Y0-6 } \\
(N=811)\end{array}$} \\
\hline & $\begin{array}{l}\mathrm{Y} 1 \\
(N=811)\end{array}$ & $\begin{array}{l}\mathrm{Y} 2 \\
(N=810)\end{array}$ & $\begin{array}{l}\text { Y3 } \\
(N=772)\end{array}$ & $\begin{array}{l}\mathrm{Y4} \\
(N=731)\end{array}$ & $\begin{array}{l}\text { Y5 } \\
(N=707)\end{array}$ & $\begin{array}{l}\text { Y6 } \\
(N=692)\end{array}$ & \\
\hline Thyroid AEs ${ }^{b}$ & $46(5.7)$ & $74(9.1)$ & $125(16.2)$ & $48(6.6)$ & $24(3.4)$ & $25(3.6)$ & 10.29 \\
\hline Hyperthyroidism $^{c}$ & $10(1.2)$ & $26(3.2)$ & $59(7.6)$ & $26(3.6)$ & $10(1.4)$ & $11(1.6)$ & 3.55 \\
\hline Hypothyroidism $^{\mathrm{d}}$ & $10(1.2)$ & $17(2.1)$ & $30(3.9)$ & $4(0.5)$ & $2(0.3)$ & $5(0.7)$ & 1.61 \\
\hline Thyroiditis $^{\mathrm{e}}$ & $6(0.7)$ & $10(1.2)$ & $14(1.8)$ & $7(1.0)$ & $6(0.8)$ & $3(0.4)$ & 1.07 \\
\hline $\begin{array}{l}\text { Decreased blood } \\
\text { TSH }\end{array}$ & $6(0.7)$ & $7(0.9)$ & $9(1.2)$ & $6(0.8)$ & $1(0.1)$ & $1(0.1)$ & 0.69 \\
\hline Goiter & $5(0.6)$ & $4(0.5)$ & $3(0.4)$ & $2(0.3)$ & $2(0.3)$ & $1(0.1)$ & 0.39 \\
\hline Increased blood TSH & $4(0.5)$ & $3(0.4)$ & $2(0.3)$ & $2(0.3)$ & $2(0.3)$ & $1(0.1)$ & 0.32 \\
\hline $\begin{array}{l}\text { Antithyroid antibody } \\
\text { positive }\end{array}$ & $2(0.2)$ & $3(0.4)$ & $4(0.5)$ & 0 & 0 & 0 & 0.20 \\
\hline $\begin{array}{l}\text { Abnormal blood } \\
\text { TSH }\end{array}$ & 0 & 0 & 0 & 0 & $1(0.1)$ & $2(0.3)$ & 0.07 \\
\hline $\begin{array}{l}\text { Abnormal thyroid } \\
\text { function test }\end{array}$ & $1(0.1)$ & $1(0.1)$ & $2(0.3)$ & 0 & 0 & $1(0.1)$ & 0.11 \\
\hline $\begin{array}{l}\text { Decreased free } \\
\text { thyroxine }\end{array}$ & $2(0.2)$ & 0 & $1(0.1)$ & 0 & 0 & 0 & 0.07 \\
\hline Decreased thyroxine & 0 & $1(0.1)$ & 0 & 0 & 0 & 0 & 0.02 \\
\hline $\begin{array}{l}\text { Increased free } \\
\text { thyroxine }\end{array}$ & 0 & $1(0.1)$ & 0 & $1(0.1)$ & 0 & 0 & 0.05 \\
\hline Toxic nodular goiter & 0 & 0 & $1(0.1)$ & 0 & 0 & 0 & 0.02 \\
\hline $\begin{array}{l}\text { Decreased free } \\
\text { triiodothyronine }\end{array}$ & 0 & $1(0.1)$ & 0 & 0 & 0 & 0 & 0.02 \\
\hline Serious thyroid AEs ${ }^{\mathrm{f}}$ & $2(0.2)$ & $5(0.6)$ & $27(3.5)$ & $6(0.8)$ & $3(0.4)$ & $1(0.1)$ & 1.03 \\
\hline Hyperthyroidism $^{c}$ & $1(0.1)$ & $2(0.2)$ & $24(3.1)$ & $5(0.7)$ & $2(0.3)$ & 0 & 0.79 \\
\hline Hypothyroidism $^{\mathrm{d}}$ & 0 & $1(0.1)$ & $1(0.1)$ & $1(0.1)$ & 0 & 0 & 0.07 \\
\hline Goiter & $1(0.1)$ & 0 & $1(0.1)$ & 0 & 0 & $1(0.1)$ & 0.07 \\
\hline $\begin{array}{l}\text { Endocrine } \\
\text { ophthalmopathy }\end{array}$ & 0 & 0 & $1(0.1)$ & 0 & 0 & 0 & 0.02 \\
\hline Thyroiditis & 0 & $1(0.1)$ & 0 & 0 & $1(0.1)$ & 0 & 0.05 \\
\hline
\end{tabular}


Table 1 continued

\begin{tabular}{|c|c|c|c|c|c|c|c|}
\hline & \multicolumn{6}{|c|}{ Incidence, $n(\%)$} & \multirow{2}{*}{$\begin{array}{l}\text { EAIR per } 100 \\
\text { patient-years }^{\mathrm{a}} \\
\text { Y0-6 } \\
(N=811)\end{array}$} \\
\hline & $\begin{array}{l}\mathrm{Y} 1 \\
(N=811)\end{array}$ & $\begin{array}{l}\mathrm{Y} 2 \\
(N=810)\end{array}$ & $\begin{array}{l}\mathbf{Y 3} \\
(\boldsymbol{N}=772)\end{array}$ & $\begin{array}{l}\text { Y4 } \\
(N=731)\end{array}$ & $\begin{array}{l}\text { Y5 } \\
(\boldsymbol{N}=707)\end{array}$ & $\begin{array}{l}\text { Y6 } \\
(N=692)\end{array}$ & \\
\hline $\begin{array}{c}\text { Increased free } \\
\text { thyroxine }\end{array}$ & 0 & $1(0.1)$ & 0 & 0 & 0 & 0 & 0.02 \\
\hline \multicolumn{8}{|c|}{$\begin{array}{l}\text { AE adverse event, EAIR exposure-adjusted incidence rate, TSH thyroid-stimulating hormone, } Y \text { year } \\
\text { a EAIR = (number of patients with first AE in the time interval)/(total follow-up duration [years] of all p } \\
\text { time interval, censoring at the time of AE for patients counted in the numerator) } \\
\text { b First occurrence of thyroid AE for a patient } \\
\text { c Hyperthyroidism denotes hyperthyroidism and Basedow's disease } \\
\text { d Hypothyroidism denotes hypothyroidism and primary hypothyroidism } \\
\text { e Thyroiditis denotes autoimmune thyroiditis, subacute thyroiditis, thyroiditis subacute, and thyroiditis } \\
\text { f First occurrence of serious thyroid AE for a patient }\end{array}$} \\
\hline
\end{tabular}

up [1.8\%], physician decision [1.2\%], death [0.7\%], lack of efficacy [0.4\%], and $\mathrm{AE}[0.1 \%]$; "other" reasons reported for $1.2 \%$ of patients). Over 6 years, $342(42 \%)$ patients experienced a first thyroid AE, with nonserious events in 298 (37\%) patients and serious events in 44 (5\%) patients. Thyroid AEs were mostly mild (grade $1,42 \%$ ) to moderate (grade 2, 56\%); few were severe (grade $3,3 \%$ ); and none were life threatening (grade 4) or fatal (grade 5; sum of percentages greater than $100 \%$ as a result of rounding). Nonserious thyroid AEs were most frequently hyperthyroidism $(42 \%$ of patients with thyroid AEs), followed by hypothyroidism (20\%) and thyroiditis (13\%). Of the 44 patients with serious thyroid AEs, 34 (77\%) had hyperthyroidism; serious goiter $(n=3 ; 7 \%)$, hypothyroidism $(n=3 ; 7 \%)$, thyroiditis $(n=2$; $5 \%)$, endocrine ophthalmopathy $(n=1 ; 2 \%)$, and increased free thyroxine $(n=1 ; 2 \%)$ were less common. Incidence of thyroid AEs peaked in year $3(16 \%)$ after receiving the first course of alemtuzumab at baseline and declined thereafter; thyroid AE rate in year 6 was $4 \%$ (Table 1). Among patients who experienced thyroid AEs through 6 years, 61 (18\%) received no thyroidrelated treatment, 281 (82\%) were managed with oral medications, $30(9 \%)$ were treated with radioiodine therapy, and 33 (10\%) were treated with thyroidectomy.
Mean time from alemtuzumab initiation to first thyroid AE was 2.5 years (median 2.3 [range 0.3-6.0]). Thyroid AEs were more prevalent in female patients, consistent with increased thyroid disorder prevalence reported in women in the general population, but other baseline characteristics were similar among patients with and without thyroid AEs [23, 24]. Baseline HRQL scores were comparable between these two groups (Table 2).

Through year 6, 302 (37\%) patients received additional courses of alemtuzumab. Among those patients who received a total of 3,4 , or 5-6 alemtuzumab courses, 41\%, 42\%, and 38\% experienced a thyroid $\mathrm{AE}$, respectively. Among those patients who did not receive any additional alemtuzumab courses, $43 \%$ had a thyroid AE over 6 years.

\section{HRQL Outcomes in Patients With and Without Thyroid AEs}

In patients without thyroid AEs or with nonserious thyroid AEs, the FAMS total score was slightly improved or similar to core study baseline at all time points through year 6 (leastsquares mean change from baseline at year 6: no thyroid AEs, 0.7; nonserious thyroid AEs, 5.1). In patients with serious thyroid AEs, the mean 
Table 2 Baseline demographics and disease characteristics

\begin{tabular}{lcc}
\hline Parameter & $\begin{array}{l}\text { With thyroid AEs } \\
(\boldsymbol{N}=\mathbf{3 4 2})\end{array}$ & $\begin{array}{l}\text { No thyroid AEs } \\
(\boldsymbol{N}=\mathbf{4 6 9})\end{array}$ \\
\hline Age, years & $34.1(8.5)$ & $33.8(8.0)$ \\
Female, $n(\%)$ & $263(76.9)$ & $267(56.9)$ \\
Caucasian, $n(\%)$ & $315(92.1)$ & $429(91.5)$ \\
EDSS score & $2.4(1.1)$ & $2.4(1.1)$ \\
Years since initial relapse & $3.3(2.6)$ & $3.4(2.4)$ \\
No. of relapses in prior year & $1.8(0.84)$ & $1.7(0.84)$ \\
No. of relapses in prior 2 years & $2.6(1.00)$ & $2.7(1.09)$ \\
Gd-enhancing lesion count & $2.36(5.1)$ & $2.28(5.9)$ \\
Patients with Gd-enhancing lesions, $n(\%)$ & $148(43.9)$ & $204(44.1)$ \\
Brain parenchymal fraction & $0.82(0.02)$ & $0.82(0.02)$ \\
FAMS (total) score & $126.8(29.39)$ & $124.9(31.54)$ \\
EQ-5D VAS score & $74.0(17.29)$ & $71.8(19.70)$ \\
SF-36 MCS score & $45.9(11.58)$ & $45.4(11.37)$ \\
SF-36 PCS score & $45.3(9.48)$ & $44.9(10.13)$ \\
\hline
\end{tabular}

Data given as mean $(\mathrm{SD})$ unless otherwise indicated

$A E$ adverse event, EDSS Expanded Disability Status Scale, EQ-5D VAS Quality of Life-5 Dimension Visual Analog Scale, FAMS Functional Assessment of Multiple Sclerosis, Gd gadolinium, MCS mental component summary, PCS physical component summary, $S D$ standard deviation, $S F-36$ 36-Item Short-Form Survey

change from baseline showed nonsignificant worsening at year $6(-5.3)$ (Fig. 2a).

HRQL outcomes measured using EQ-5D VAS were slightly improved or similar to baseline in all subgroups of alemtuzumab-treated patients except that at year 6 scores were significantly lower than baseline in patients with serious thyroid AEs (least-squares mean change from baseline at year 6: no thyroid AEs, 2.0; nonserious thyroid AEs, 3.0; serious thyroid AEs, - 6.8) (Fig. 2b).

SF-36 MCS scores remained above baseline at most time points in patients who experienced no thyroid AEs or nonserious thyroid AEs, but were not significantly different from baseline in patients with serious thyroid AEs (least-squares mean change from baseline at year 6: no thyroid AEs, 0.6; nonserious thyroid AEs, 1.6; serious thyroid AEs, - 2.8) (Fig. 2c). SF-36 PCS scores were slightly improved or similar to baseline throughout the study (least-squares mean change from baseline at year 6: no thyroid AEs, 0.8; nonserious thyroid AEs, 1.0; serious thyroid AEs, 1.1) (Fig. 2d). The occurrence of nonserious thyroid AEs throughout the study did not have an impact on SF-36 outcomes at study end (i.e., at year 6); similar percentages of patients without thyroid AEs (MCS, 74\%; PCS, $82 \%$ ), and with nonserious thyroid AEs (both MCS and PCS, 81\%) had improved or stable SF36 scores over 6 years (Fig. 3). The subgroup with serious thyroid AEs had more patients with worsened MCS scores (37\%), but this was less apparent for PCS outcomes (23\%).

\section{HRQL Worsening Was Associated with Thyroidectomy and Radioiodine Treatment for Thyroid AEs}

Among patients with serious thyroid AEs $(n=44), 24$ experienced FAMS score worsening 
(a) FAMS (total) score

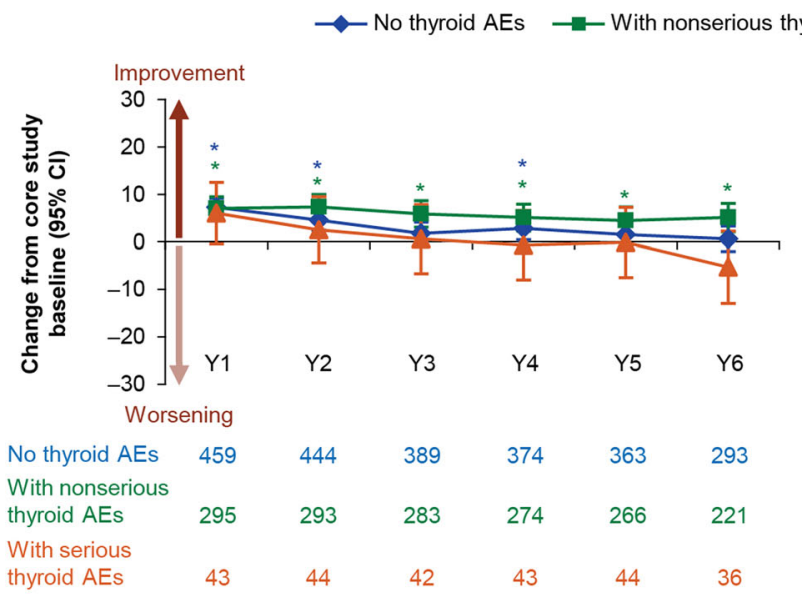

(b) EQ-5D VAS score

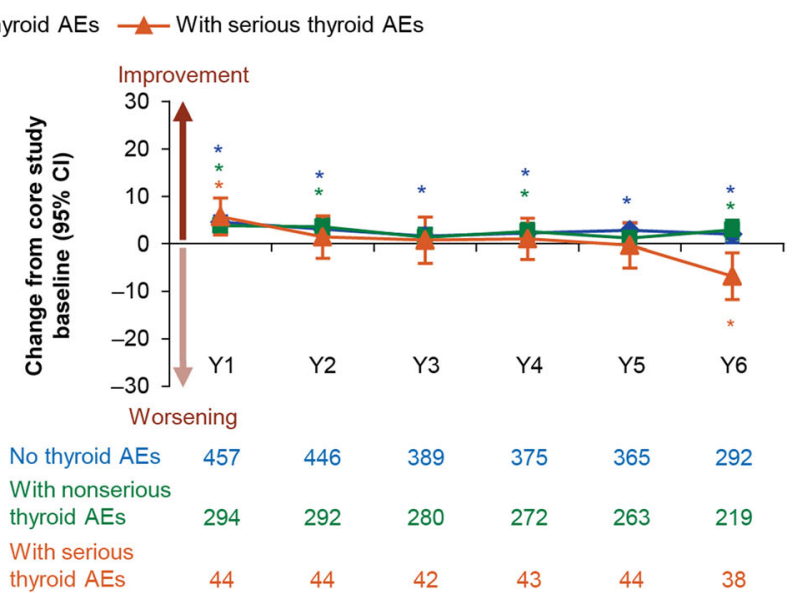

(c) SF-36 MCS score

(d) SF-36 PCS score

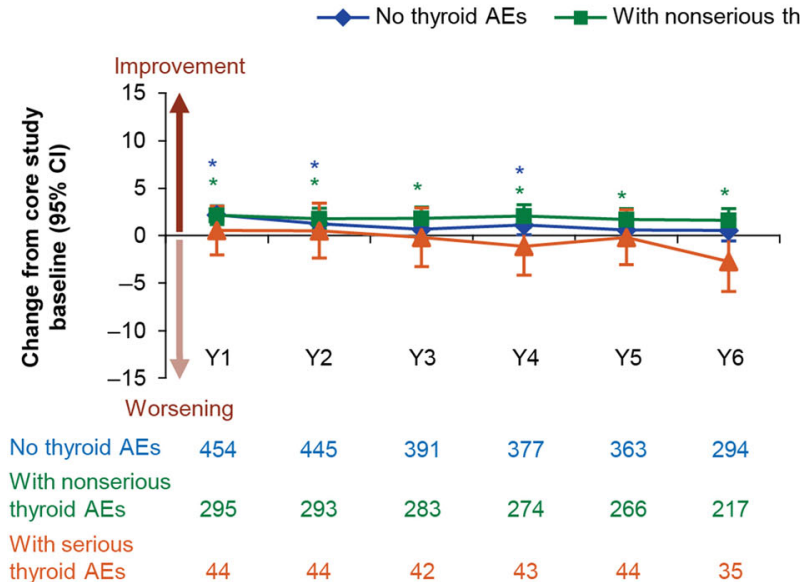

Fig. 2 HRQL changes from core study baseline over 6 years in a FAMS (total) score, b EQ-5D VAS score, c SF-36 MCS score, and d SF-36 PCS score. Data are leastsquares means $(95 \% \mathrm{CI})$. Higher scores represent better HRQL. ${ }^{*} p<0.05$ for comparison of postbaseline time point with baseline. AE adverse event, CI confidence

over 6 years. Of these, $71 \%$ had either thyroidectomy $(n=15)$ or radioiodine therapy $(n=2)$. A similar relationship between worsened HRQL and these treatments for thyroid AEs was observed for EQ-5D VAS (20 patients worsened, $75 \%$ with thyroidectomy $[n=13]$ or radioiodine treatment $[n=2])$, SF-36 MCS (25 patients worsened, $72 \%$ with thyroidectomy $[n=17]$ or radioiodine treatment $[n=1])$, and SF-36 PCS (19 patients worsened, 74\% with

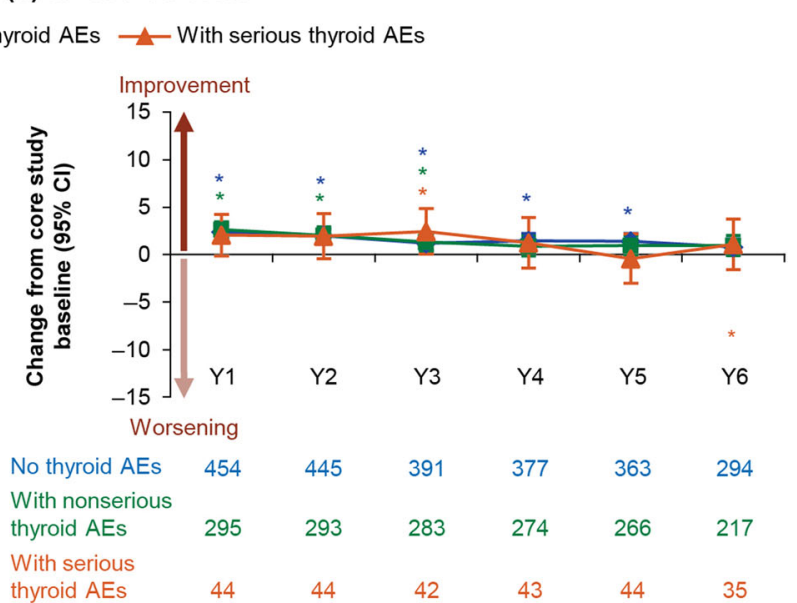

interval, EQ-5D VAS Quality of Life-5 Dimension Visual Analog Scale, FAMS Functional Assessment of Multiple Sclerosis, HRQL health-related quality of life, MCS mental component summary, PCS physical component summary, SF-36 36-Item Short-Form Survey, Y year

thyroidectomy $[n=12]$ or radioiodine treatment $[n=2])$.

\section{HRQL During Peak Onset of Thyroid AEs}

In patients who experienced a thyroid $\mathrm{AE}$ (nonserious or serious, $n=125$ ) or serious thyroid $\mathrm{AE}(n=27)$ in year 3 , there were minimal changes in HRQL outcomes from the end of year 2 to the end of year 3 , during the time of 


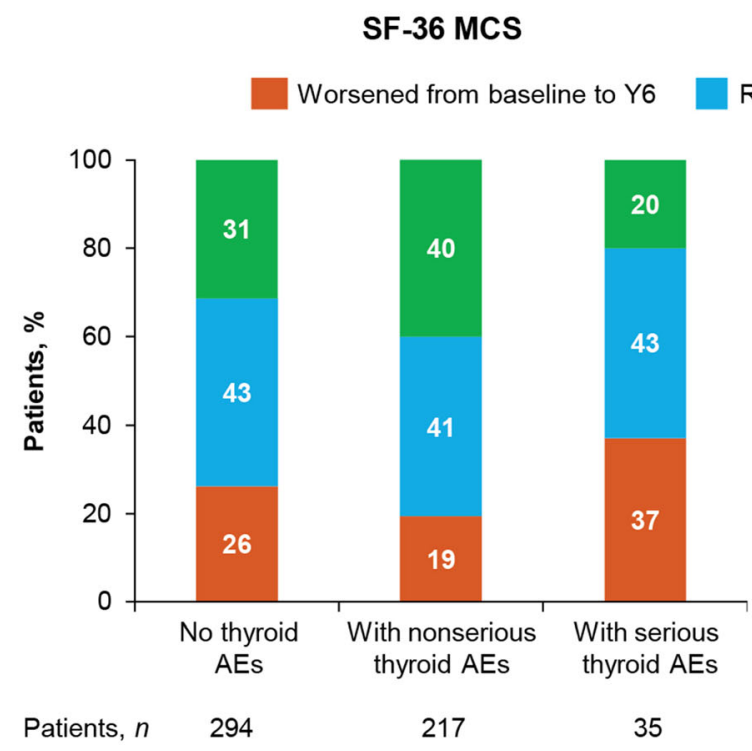

Fig. 3 Improvement, stability, and worsening in SF-36 scores from core study baseline to year 6. Clinically meaningful worsening and improvement were defined as at least a 5-point increase and decrease from baseline

AE onset (Fig. 4). HRQL outcomes remained improved or similar to core study baseline at the end of year 3 in most patients with any thyroid $\mathrm{AE}$ (least-squares mean change from baseline: FAMS, 5.8; EQ-5D VAS, 2.8; SF-36 MCS, 1.8; SF36 PCS, 1.2).

\section{DISCUSSION}

The present study demonstrated that over 6 years, mean HRQL outcomes measured by FAMS, EQ-5D VAS, and SF-36 generally remained slightly improved or similar to baseline in most patients who developed thyroid AEs after initiating alemtuzumab treatment. HRQL outcomes were similar in patients with nonserious thyroid AEs and those without thyroid AEs, indicating that nonserious thyroid AEs did not adversely affect patients' perceptions of their physical and mental health. Because the majority of patients had either no thyroid AEs or nonserious thyroid AEs, the magnitude of HRQL change in these subgroups is consistent with previous studies demonstrating HRQL improvement in the overall CARE-MS I and II populations after treatment with alemtuzumab,
SF-36 PCS

Remained stable Improved from baseline to $\mathrm{Y} 6$

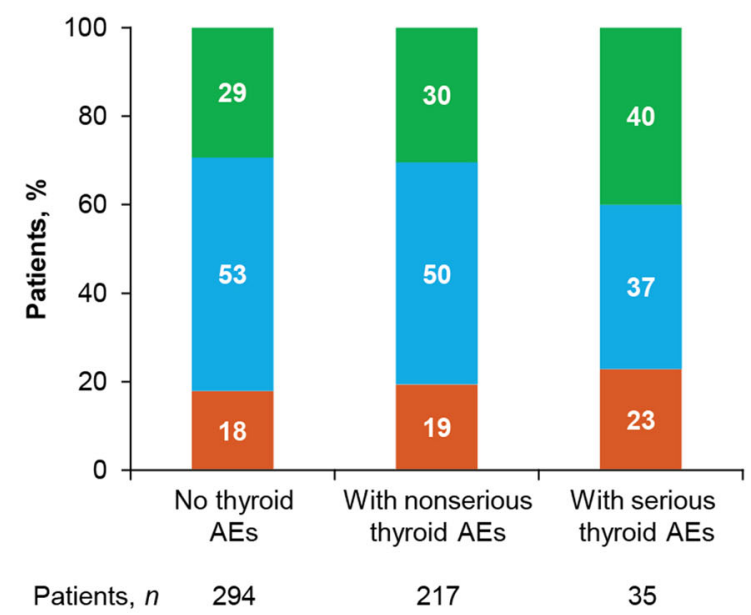

norm-based scores, respectively. AE adverse event, MCS mental component summary, PCS physical component summary, SF-36 36-Item Short-Form Survey, Y year

which stabilized over 6 years [7-9]. These HRQL findings likely reflect the efficacy of alemtuzumab, in which $78-81 \%$ of patients show stable or improved disability on the Expanded Disability Status Scale (EDSS) over 6 years $[5,6]$.

Patients with serious thyroid AEs showed worsened mean HRQL scores at later time points in the study at the population level, but only about half of the individuals with serious thyroid AEs had worsened scores. Most individuals with worsened HRQL scores were treated with radioiodine therapy or thyroidectomy. It is possible that worsened HRQL was a result of these patients having more or prolonged symptoms since these treatments are usually reserved for severe or refractory cases. Other studies in non-alemtuzumab-treated patients have shown that thyroid-related symptoms contribute to worsening of HRQL [25, 26]. Analysis of HRQL during the peak year of thyroid $\mathrm{AE}$ onset showed little to no worsening of HRQL in CARE-MS patients during serious thyroid $\mathrm{AE}$ onset, supporting the idea that worsened HRQL results from persistent thyroid symptoms over an extended period of time. Additionally, worsened HRQL may be related to 
(a) FAMS (total) score

(b) EQ-5D VAS score

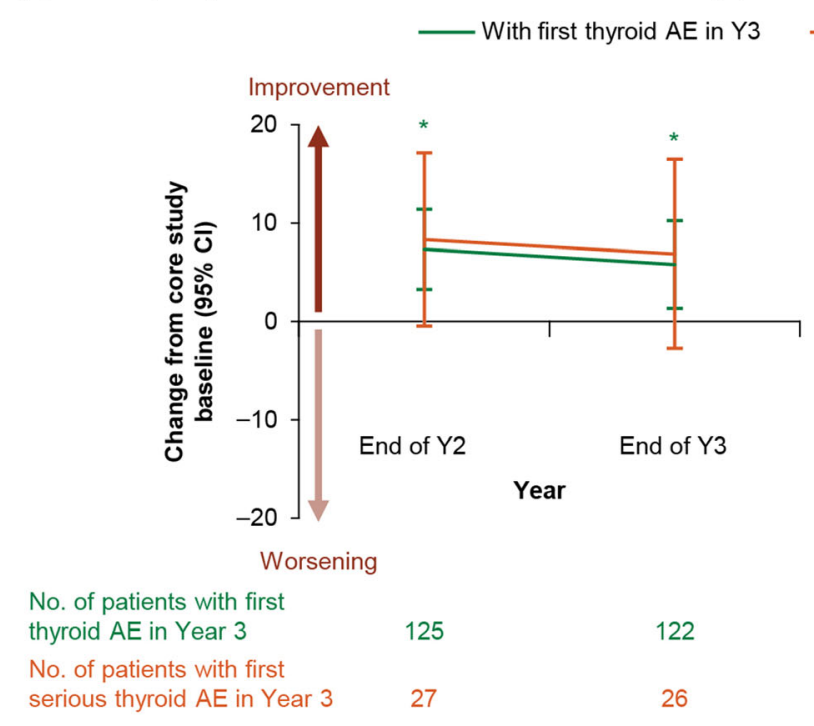

With first serious thyroid $A E$ in $Y 3$

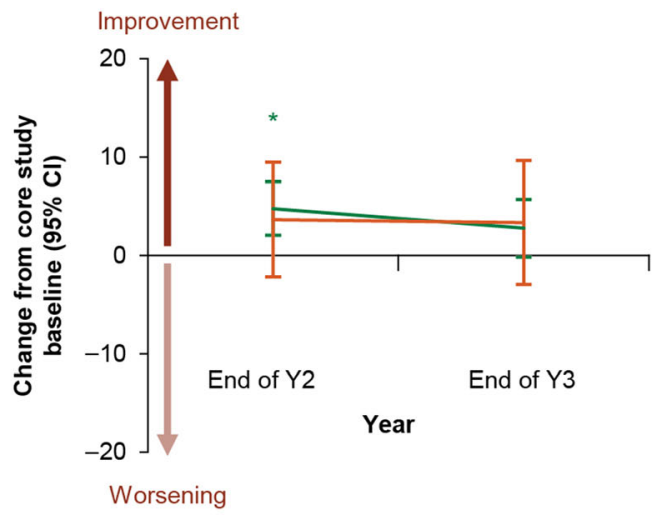

(c) SF-36 MCS score

(d) SF-36 PCS score
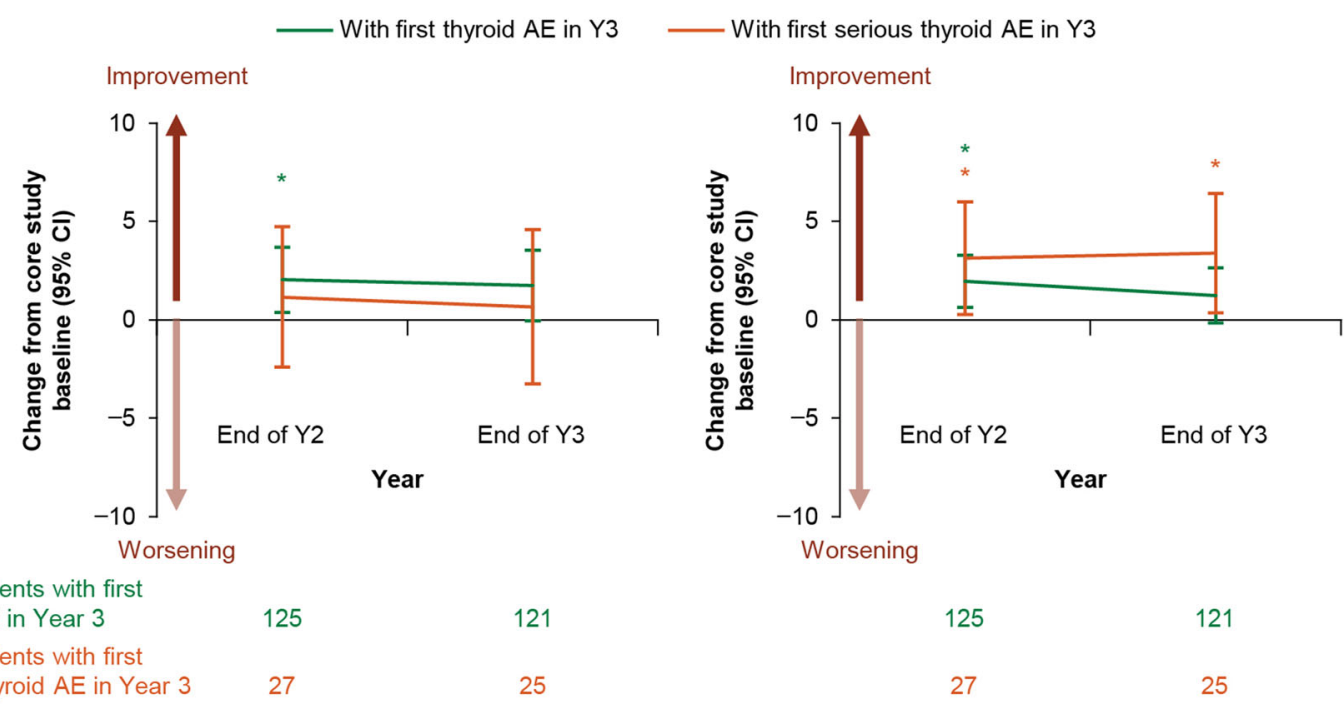

Fig. 4 HRQL changes during the time of onset of any thyroid AE or serious thyroid AE in a FAMS (total) score, b EQ-5D VAS score, c SF-36 MCS score, and d SF-36 PCS score. Analyses restricted to patients with thyroid AEs in year 3, comparing patients before onset of thyroid $\mathrm{AE}$ (end of year 2) and during or after onset of thyroid $\mathrm{AE}$ (end of year 3). Data are least-squares means (95\% CI).

the more invasive thyroid treatments these patients ultimately underwent. Nevertheless, patients with serious thyroid AEs overall had only subtle worsening in mean HRQL scores. These results indicate that for many patients
${ }^{*} p<0.05$ for comparison of postbaseline time point with baseline. AE adverse event, CI confidence interval, EQ-5D VAS Quality of Life-5 Dimension Visual Analog Scale, FAMS Functional Assessment of Multiple Sclerosis, HRQL health-related quality of life, MCS mental component summary, PCS physical component summary, SF36 36-Item Short-Form Survey, Y year

with serious thyroid AEs, net HRQL impact was likely minimal.

Considering the frequency of thyroid AEs, healthcare practitioners and patients may be concerned that these AEs are a tradeoff when 
treating with alemtuzumab, replacing one autoimmune condition with another. However, studies have shown that patients with MS have large decreases (at least 10 points) across all domains of the SF-36 compared with general population norms [27]; the ability of alemtuzumab to improve or stabilize HRQL over the long term, even in the presence of thyroid AEs, suggests positive benefit-risk for this tradeoff.

Thyroid function was tested regularly during the CARE-MS studies. Timely detection and treatment of thyroid dysfunction in alemtuzumab-treated patients may be key for preserving HRQL gains associated with clinical and MRI improvements. The monitoring protocol used in the CARE-MS studies later formed the basis for the postmarketing Risk Evaluation and Mitigation Strategy in the USA and the Risk Management Plan in Europe to ensure adequate follow-up of patients in real-world settings. The minimal impact of thyroid AEs on HRQL in the CARE-MS studies implies that the monitoring program successfully mitigated thyroid AE risk.

The present study has several limitations. First, SF-36 and EQ-5D VAS are generic measures and, therefore, may not be as sensitive to HRQL changes as the disease-specific FAMS. Second, patients had knowledge of their treatment assignment and thyroid AEs, which may have influenced their HRQL responses. Third, HRQL outcomes might also be influenced by unknown factors or individual differences in perception of disease. Finally, HRQL assessments were conducted annually, so the temporal relationship between HRQL assessment and the peak of thyroid $\mathrm{AE}$ impact (either $\mathrm{AE}$ onset or during thyroidectomy or radioiodine therapy) is variable between patients.

\section{CONCLUSIONS}

This is the first study to evaluate the impact of autoimmune thyroid AEs on HRQL in alemtuzumab-treated patients with relapsing-remitting MS (RRMS). Autoimmune thyroid AEs had minimal impact on HRQL outcomes for most alemtuzumab-treated patients with RRMS; worsened HRQL scores were associated with thyroidectomy or radioiodine treatment. These data provide context to patient experience with alemtuzumab-related thyroid AEs and can aid in therapeutic decision-making.

\section{ACKNOWLEDGEMENTS}

The authors and Sanofi would like to thank the patients for their participation in the trials, as well as the CARE-MS I and CARE-MS II Steering Committees and the CAMMS03409 Investigators.

Funding. The study was supported by Sanofi and Bayer HealthCare Pharmaceuticals (Leverkusen, Germany). The sponsor was involved in design and conduct of the study; collection, management, analysis, and interpretation of the data; and preparation, review, or approval of the manuscript. The journal's Rapid Service Fee was provided by Sanofi.

Medical Writing, Editorial and Other Assistance. The manuscript was reviewed for scientific accuracy by Darren P. Baker, PhD, Ericka M. Bueno, PhD, and Jonathan Valenzano, PharmD, of Sanofi (Cambridge, MA, USA). Additional statistical support was provided by Cytel, Inc. (Cambridge, MA, USA). Editorial support was provided by Valerie P. Zediak, PhD, and Panos Xenopoulos, PhD, of Eloquent Scientific Solutions (Philadelphia, PA, USA), and was funded by Sanofi.

Authorship. All authors meet the International Committee of Medical Journal Editors (ICMJE) criteria for authorship for this article. All authors had full access to the study data and take responsibility for the integrity of the data and the accuracy of the data analysis, and have given their approval for this version to be published.

Disclosures. Antonio Bertolotto has received consulting fees from Biogen and Genzyme; and lecture fees from Biogen, Merck, Novartis, Roche, Sanofi, and Teva. Antonio Bertolotto is also the journal's Editor-in-Chief. Rafael Arroyo has served as an advisory board participant and 
received speaking fees from Almirall, Bayer, Biogen, Merck, Novartis, Roche, Sanofi, and Teva. Elisabeth G. Celius has served on advisory boards and/or received speaking honoraria from Biogen, Genzyme, Merck, Novartis, Roche, and Teva; and unrestricted research grants from Genzyme and Novartis. Giancarlo Comi has received consulting fees from Actelion, BayerSchering, Merck Serono, Novartis, Sanofi, and Teva; and lecture fees from Bayer-Schering, Biogen Dompé, Merck Serono, Novartis, Sanofi, Serono Symposia International Foundation, and Teva. Eva Kubala Havrdova has received honoraria and grant support from Actelion, Biogen, Merck Serono, Novartis, Receptos, Roche, Sanofi, and Teva; and support from the Ministry of Education of the Czech Republic, project PROGRES Q27/LF1. William David Honeycutt has received consulting fees, speaker honoraria, and/or research support from Actelion, Alkermes, Biogen-Idec, Celgene, EMD Serono, Mallinckrodt, MedImmune, Novartis, Roche, Sanofi, and Teva. Samuel F. Hunter has received speaker honoraria, consulting agreements, and grant/research financial support from AbbVie, Acorda, Actelion, Adamas, Alkermes, Avanir, Bayer, Biogen Idec, Novartis, Osmotica, Mallinckrodt, Roche, Sanofi, Synthon, and Teva. Guillermo Izquierdo has received speaking and advisory fees from Almirall, Bayer, Biogen, Merck Serono, Novartis, Roche, Sanofi, and Teva. Barbara Kornek has received consulting and/or speaking fees from Bayer, Biogen, Celgene, Merck, Novartis, Roche, Sanofi, and Teva. Tamara Miller has received speaking and/ or consulting fees from Acorda, Allergan, Amgen, Biogen, Genentech, Genzyme, Lilly, Mallinckrodt, Novartis, Reven, Sanofi, and Teva; and research support from Adamas, Allergan, Biogen, Elan, EMD Serono, Genentech, Ipsen, Lilly, Novartis, Ono, Sun Pharma, and Teva. Dimos D. Mitsikostas has received speaking and/or consulting fees from Allergan, Amgen, Bayer, Cefaly, Eli Lilly, ElectroCore, Genesis Pharma, Merz, Mylan, Novartis, Roche, Sanofi, and Teva; and grant research support from Biogen and Sanofi. Barry A. Singer has received speaking and/or consulting from AbbVie, Acorda, Alexion, Biogen, Celgene, EMD Serono, Genentech, Novartis, Roche, Sanofi, Teva, and
TG Therapeutics; and research grant support from AbbVie, Alkermes, Biogen, MedImmune, Novartis, Roche, and Sanofi. Tjalf Ziemssen has received consulting and/or speaking fees from Almirall, Bayer, Biogen, Merck, Novartis, Roche, Sanofi, and Teva; and grant/research support from Biogen, Novartis, Sanofi, and Teva. Luke Chung, Nadia Daizadeh, Salman Afsar, and Lobat Hashemi are employees of Sanofi. Peter Senior has received consulting and/or speaking fees from Abbott, AstraZeneca, Bayer, Boehringer Ingelheim, Eli Lilly, Janssen, Novartis, Novo Nordisk, Sanofi, Servier, and Valeant; and grant research support from Novo Nordisk, Prometic, and Servier.

Compliance with Ethics Guidelines. The CARE-MS I and II studies and the CARE-MS extension study were registered with ClinicalTrials.gov (NCT00530348, NCT00548405, and NCT00930553, respectively). The studies were conducted in accordance with the ethical principles outlined in the Declaration of Helsinki. All procedures were approved by local institutional ethics review boards of participating sites (see Table $S 1$ in the electronic supplementary material for details). Patients provided written informed consent.

Data Availability. Qualified researchers may request access to patient-level data and related study documents including the clinical study report, study protocol with any amendments, blank case report form, statistical analysis plan, and data set specifications. Patientlevel data will be anonymized and study documents will be redacted to protect the privacy of trial participants. Further details on Sanofi's data-sharing criteria, eligible studies, and process for requesting access can be found at https://www.clinicalstudydatarequest.com.

Open Access. This article is licensed under a Creative Commons Attribution-NonCommercial 4.0 International License, which permits any non-commercial use, sharing, adaptation, distribution and reproduction in any medium or format, as long as you give appropriate credit to the original author(s) and the source, provide a link to the Creative 
Commons licence, and indicate if changes were made. The images or other third party material in this article are included in the article's Creative Commons licence, unless indicated otherwise in a credit line to the material. If material is not included in the article's Creative Commons licence and your intended use is not permitted by statutory regulation or exceeds the permitted use, you will need to obtain permission directly from the copyright holder. To view a copy of this licence, visit http:// creativecommons.org/licenses/by-nc/4.0/.

\section{REFERENCES}

1. Berger JR. Functional improvement and symptom management in multiple sclerosis: clinical efficacy of current therapies. Am J Manag Care. 2011;17(Suppl 5):S146-53.

2. Sorensen PS. Balancing the benefits and risks of disease-modifying therapy in patients with multiple sclerosis. J Neurol Sci. 2011;311(Suppl 1):S29-34.

3. Cohen JA, Coles AJ, Arnold DL, et al. Alemtuzumab versus interferon beta $1 \mathrm{a}$ as first-line treatment for patients with relapsing-remitting multiple sclerosis: a randomised controlled phase 3 trial. Lancet. 2012;380(9856):1819-28.

4. Coles AJ, Twyman CL, Arnold DL, et al. Alemtuzumab for patients with relapsing multiple sclerosis after disease-modifying therapy: a randomised controlled phase 3 trial. Lancet. 2012;380(9856): 1829-39.

5. Fox EJ, Brassat D, Alroughani R, et al. Alemtuzumab provides durable efficacy over 6 years in patients with active relapsing-remitting multiple scle.rosis and an inadequate response to prior therapy in the absence of continuous treatment (CARE-MS II). Neurology. 2017;88(Suppl 16):S24. 006.

6. Singer B, Coles AJ, Boyko AN, et al. Improvements in clinical outcomes with alemtuzumab in treatment-naive patients with active relapsing-remitting multiple sclerosis are durable over 6 years in the absence of continuous treatment (CARE-MS I). Neurology. 2017;88(Suppl 16):S24.005.

7. Arroyo Gonzalez R, Kita M, Crayton $\mathrm{H}$, et al. Alemtuzumab improves quality-of-life outcomes compared with subcutaneous interferon beta-1a in patients with active relapsing-remitting multiple sclerosis. Mult Scler. 2017;23(10):1367-76.
8. Arroyo R, Bury DP, Guo JD, et al. Impact of alemtuzumab on health-related quality of life over 6 years in CARE-MS II trial extension patients with relapsing-remitting multiple sclerosis. Mult Scler. 2019. https://doi.org/10.1177/1352458519849796.

9. Arroyo Gonzalez R, Guo J, Zhang W, et al. Alemtuzumab improves patient-reported quality of life outcomes in patients with relapsing-remitting multiple sclerosis: results from the CARE-MS I extension study. Neurology. 2018;90(P6):380.

10. Coles AJ, Cohen JA, Fox EJ, et al. Alemtuzumab CARE-MS II 5-year follow-up: efficacy and safety findings. Neurology. 2017;89(11):1117-26.

11. LEMTRADA $^{\circledR}$ (alemtuzumab) [Prescribing Information] 2019. Cambridge: Genzyme Corporation.

12. Havrdova E, Arnold DL, Cohen JA, et al. Alemtuzumab CARE-MS I 5-year follow-up: durable efficacy in the absence of continuous MS therapy. Neurology. 2017;89(11):1107-16.

13. LEMTRADA [Summary of Product Characteristics] April 2019. Diegem: Sanofi Belgium.

14. Twyman C, Oyuela P, Palmer J, Margolin D, Dayan C. Thyroid autoimmune adverse events in patients treated with alemtuzumab for relapsing-remitting multiple sclerosis: four-year follow-up of the CAREMS studies. Neurology. 2014;82(P2):199.

15. Devonshire V, Phillips R, Wass H, Da Roza G, Senior P. Monitoring and management of autoimmunity in multiple sclerosis patients treated with alemtuzumab: practical recommendations. J Neurol. 2018;265(11):2494-505.

16. Decallonne B, Bartholome E, Delvaux V, et al. Thyroid disorders in alemtuzumab-treated multiple sclerosis patients: a Belgian consensus on diagnosis and management. Acta Neurol Belg. 2018;118(2): 153-9.

17. Cancer Therapy Evaluation Program. Common terminology criteria for adverse events v3.0 (CTCAE). National Cancer Institute; [updated 2006 Aug 9; cited 2020 Feb 4]. https://ctep.cancer.gov/ protocolDevelopment/electronic_applications/ docs/ctcaev3.pdf.

18. Cella DF, Dineen K, Arnason B, et al. Validation of the functional assessment of multiple sclerosis quality of life instrument. Neurology. 1996;47(1):129-39.

19. Brooks R. EuroQol: the current state of play. Health Policy. 1996;37(1):53-72.

20. Ware JE Jr, Sherbourne CD. The MOS 36-item shortform health survey (SF-36). I. Conceptual 
framework and item selection. Med Care. 1992;30(6):473-83.

21. Ware JE Jr, Kosinski M, Bjorner JB, Turner-Bowker DM, Gandek B, Maruish ME. User's Manual for the $36 \mathrm{v} 2^{\circledR}$ Health Survey. 2nd ed. Lincoln: Quality Metric; 2007.

22. Rudick RA, Miller D, Hass S, et al. Health-related quality of life in multiple sclerosis: effects of natalizumab. Ann Neurol. 2007;62(4):335-46.

23. Stahlman S, Oh GT. Thyroid disorders, active component, U.S. Armed Forces, 2008-2017. MSMR. 2018;25(12):2-9.

24. Khattak RM, Ittermann $\mathrm{T}$, Nauck $\mathrm{M}$, Below $\mathrm{H}$, Volzke H. Monitoring the prevalence of thyroid disorders in the adult population of Northeast Germany. Popul Health Metr. 2016;14:39.
25. Shivaprasad C, Boppana R, Kolly A, Pullikal A, Goel A, Dwarkanath C. Impairment of health-related quality of life among Indian patients with hypothyroidism. Indian $\mathrm{J}$ Endocrinol Metab. 2018;22(3):335-8.

26. Elberling TV, Rasmussen AK, Feldt-Rasmussen U, Hording $\mathrm{M}$, Perrild $\mathrm{H}$, Waldemar G. Impaired health-related quality of life in Graves' disease. A prospective study. Eur J Endocrinol. 2004;151(5): 549-55.

27. Riazi A, Hobart J, Lamping D, et al. Using the SF-36 measure to compare the health impact of multiple sclerosis and Parkinson's disease with normal population health profiles. J Neurol Neurosurg Psychiatry. 2003;74(6):710-4. 